\title{
É Imperiosa a Indicação de Estudo Eletrofisiológico Invasivo em Pacientes Sobreviventes de Parada Cardíaca?
}

\author{
José Tarcísio Medeiros de Vasconcelos, Silas dos Santos Galvão Filho, Cecília Monteiro Boya Barcellos
}

São Paulo, SP

\begin{abstract}
A indicação de estudo eletrofisiológico invasivo em sobreviventes de parada cardíaca é uma prática rotineira e classicamente recomendada pelas associações médicas ${ }^{1,2}$. Contudo, essa linha de conduta vem sendo alvo de discussão, após o advento dos cardioversores desfibriladores implantáveis ${ }^{3,4}$. O impacto positivo causado por essas próteses na mortalidade total e súbita nessa população trouxe uma natural tendência à busca de simplificação na abordagem terapêutica desses pacientes. Nessa tendência, considerando que cerca de $95 \%$ dos casos de parada cardíaca são decorrentes de fibrilação ventricular ${ }^{5}$, os elevados índices de recorrência do quadro após um evento inicial abortado ${ }^{6}$ e a eficácia dos cardioversores desfibriladores implantáveis em sua prevenção secundária, quando comparada a outras estratégias de tratamento ${ }^{7-10}$, tornaram importante reavaliar o papel do estudo eletrofisiológico invasivo, como método propedêutico e auxiliar terapêutico nas vítimas de parada cardíaca recuperada.
\end{abstract}

\section{Emprego do estudo eletrofisiológico invasivo em sobreviventes de parada cardíaca}

O fundamento lógico para justificar o emprego do estudo eletrofisiológico invasivo nos sobreviventes de parada cardíaca é o de estabelecer o diagnóstico do distúrbio do ritmo cardíaco causador do evento e, conseqüentemente, orientar o tratamento individualizado mais adequado. Condições de significado clínico e terapêutico distintos podem ser identificadas nessa investigação: a) bradiarritmias: o estudo eletrofisiológico invasivo pode identificar a presença de uma disfunção sinusal ou um distúrbio grave da condução atrioventricular, cujo tratamento envolveria simplesmente o implante de um marcapasso definitivo; b) taquicar-

Clínica de Ritmologia Cardíaca do Hospital Beneficência Portuguesa de São Paulo Correspondência: José Tarcísio Medeiros de Vasconcelos - Rua Maestro Cardim, 1041 - 01323-001 - São Paulo, SP - E-mail: jtarr@uol.com.br

Recebido para publicação em 1/10/01

Aceito em 21/1/02 dias supraventriculares: uma síndrome de pré-excitação ou qualquer forma de taquicardia supraventricular pode ser diagnosticada. A ablação por cateter permitiria uma solução definitiva para o problema; c) taquicardias ventriculares monomórficas ou polimórficas, ou mesmo a fibrilação ventricular podem ser induzidas e indiretamente implicadas na gênese do evento clínico (parada cardíaca). Tal achado abriria um leque de possibilidades terapêuticas, como o tratamento farmacológico guiado por estimulação, o simples tratamento farmacológico empírico, a ablação por cateter, a ablação cirúrgica, ou o implante de um cardioversor desfibrilador implantável.

A despeito dessas considerações, é importante frisar que a morte súbita é uma síndrome complexa, cujos mecanismos são pouco compreendidos. As informações fornecidas pelo estudo eletrofisiológico invasivo, naqueles indivíduos que sobrevivem ao evento, permitem no máximo que se suponha seu mecanismo, frente a um ou mais achados, $o$ que não necessariamente corresponde àquilo que ocorre na prática.

\section{Bradiarritmias}

A associação entre bradiarritmias e morte súbitaé motivo de controvérsia de natureza epidemiológica. Estudos envolvendo documentação de morte súbita por meio de monitorização eletrocardiográfica pelo sistema Holter indicam que, em 16 a 20\% dos casos, uma bradicardia corresponde ao evento final ${ }^{11,12}$. A depressão da função sinusal parece ser mais freqüente do que o bloqueio atrioventricular ${ }^{11}$. Em um levantamento de 225 casos de morte súbita publicado por Myerburg e cols. ${ }^{13}$, envolvendo indivíduos que receberam atendimento médico por ocasião da ocorrência do evento, foram observadas bradiarritmias, definidas por assistolia, bradicardia sinusal ou ritmo idioventricular, em $27 \%$ dos pacientes. Em portadores de insuficiência cardíaca congestiva severa esses índices são mais elevados. Luu e cols. ${ }^{14}$ avaliaram 21 casos de morte súbita em indivíduos, que aguardavam transplante cardíaco, constatando que em apenas oito $(38 \%)$ pacientes, taquicardias ventriculares correspon- 
deram ao evento final. Em 13 (62\%) casos, a parada cardíaca foi atribuída a assistolia ou dissociação eletromecânica, sendo que em sete não foram identificadas causas secundárias para o evento; nos seis pacientes remanescentes, fatores precipitantes, como isquemia miocárdica, tromboembolismo pulmonar e alterações metabólicas foram constatadas. Quando se analisam esses dados de forma crítica, conclui-se que esses valores estão superestimados. As documentações eletrocardiográficas de morte súbita pelo sistema Holter incorporam amostras selecionadas de pacientes, que não são representativas da população geral. Ainda, a documentação de uma assistolia por ocasião do atendimento a uma vítima de morte súbita pode simplesmente ser demonstrativa de uma demora na assistência médica adequada; a assistolia seria apenas o estágio final de uma situação de fibrilação ventricular e hipóxia prolongadas. Tais observações são relevantes quando se analisam os achados eletrocardiográficos em vítimas de morte súbita, considerados de acordo com o tempo de atendimento médico; quando o tempo decorrido entre a ocorrência do evento e a assistência médica é inferior a 4min, a assistolia é encontrada em apenas $5 \%$ dos casos, atingindo $25 \%$ quando esse tempo é superior a $16 \mathrm{~min}^{5}$. Essa constatação, em tese, contribui para justificar os baixos índices de sobrevida, quando a assistolia é o achado eletrocardiográfico em vítimas de morte súbita, que não ultrapassam $15 \%{ }^{12,13}$.

O papel do estudo eletrofisiológico invasivo na identificação de bradiarritmias, que estejam envolvidas na gênese da parada cardíaca recuperada, não pode ser estabelecido à luz das evidências atuais. A constatação de anormalidades da função sinusal e/ou da condução atrioventricular não necessariamente permite estabelecer uma relação de causa e efeito, considerando o fato que as bradiarritmias são responsáveis pela minoria dos casos de morte súbita. Desse modo, achados dessa natureza não podem determinar com segurança a melhor conduta terapêutica a ser adotada. Ainda, algumas entidades potencialmente responsáveis por morte súbita assistólica são transitórias, como é o caso das anormalidades de controle neural ${ }^{15}$, não podendo ser identificadas no estudo eletrofisiológico invasivo.

\section{Taquicardias supraventriculares}

As taquicardias supraventriculares podem estar implicadas na morte súbita cardíaca tanto como fator causal direto como indireto, complicando uma doença cardíaca coexistente ${ }^{16-18}$. Em um levantamento de 290 casos de parada cardíaca abortada, publicado por Wang e cols. ${ }^{18}$, taquicardias supraventriculares que deterioravam em fibrilação ventricular corresponderam ao fator etiológico em $13(4,5 \%)$ pacientes, identificada por documentação eletrocardiográfica ou por inferência com base nos achados de estudo eletrofisiológico. Foram constatadas taquicardias atriais primárias com resposta ventricular elevada na ausência ou presença de síndromes de pré-excitação, taquicardia atrioventricular recíproca da síndrome de Wolff-Parkinson-White e mesmo taquicardias por reentrada nodal. O tratamento medicamen- toso ou definitivo por ablação por cateter ou cirúrgica evitou a recorrência de fibrilação ventricular em todos os pacientes, em um seguimento médio de 42 meses. É digno de menção nessa série que, em outros 27 pacientes, no estudo eletrofisiológico invasivo foram induzidas tanto taquicardias supraventriculares como taquicardias ventriculares; como as taquicardias ventriculares cursavam com menor tolerabilidade hemodinâmica, os autores concluíram que estas, e não as primeiras, foram o fator causador da parada cardíaca nesses casos. Achado que põe em questão o significado clínico das taquicardias supraventriculares induzidas em laboratório, nos indivíduos sobreviventes de parada cardíaca não documentada.

\section{Taquicardias ventriculares}

Como já discutido, a fibrilação ventricularé, de longe, o principal distúrbio do ritmo cardíaco identificado por ocasião de uma parada cardíaca. Embora as documentações eletrocardiográficas de morte súbita pelo sistema Holter indiquem que, na maioria desses casos, a fibrilação ventricular tenha como precursora uma taquicardia ventricular monomórfica ${ }^{11}$, acredita-se que aqui, mais uma vez, esses resultados estejam superestimados, visto que esses estudos foram conduzidos em pacientes selecionados ${ }^{4}$. Na realidade, as evidências obtidas de séries envolvendo populações não-específicas de pacientes indicam que o índice dos casos em que uma taquicardia ventricular monomórfica corresponde ao evento precursor da parada cardíaca não atinge $10 \%^{19,20}$.

A aplicabilidade do estudo eletrofisiológico em sobreviventes de parada cardíaca decorrente, ou supostamente decorrente, de taquicardias primariamente ventriculares tem, em tese, dois objetivos. O primeiro é de diagnóstico: tratando-se de uma parada cardíaca não documentada, a indução de taquicardia ventricular monomórfica sustentada, taquicardia ventricular polimórfica, ou fibrilação ventricular permite que se conclua, indiretamente, que aquela taquicardia induzida corresponde ao fator responsável pelo evento clínico. A não-inductibilidade de qualquer taquicardia permite inferir que o evento clínico não tenha sido motivado por arritmia ventricular. Tratando-se de uma parada cardíaca documentada por fibrilação ventricular, a indução de taquicardia ventricular monomórfica permite concluir que a fibrilação ventricular tenha sido motivada pela taquicardia induzida. O segundo objetivo é terapêutico: a indução de qualquer forma de taquicardia pode orientar o tratamento farmacológico, guiado por estimulação através de estudos seriados, buscando-se a supressão da indução. A terapia farmacológica empírica pode ser outra estratégia terapêutica frente a uma taquicardia induzida, previamente desconhecida. Taquicardias ventriculares monomórficas induzidas podem ser mapeadas e tratadas por ablação por cateter ou cirúrgica. A indução de taquicardias não-mapeáveis pode servir como achado de orientação para indicação de implante de um cardioversor desfibrilador implantável.

Como já citado, porém, todas essas considerações são de natureza teórica. A análise dos diferentes estudos sobre o tema, em uma perspectiva crítica, demonstra que, na práti- 
ca, a maior parte dessas inferências não encontram respaldo. Em sobreviventes de parada cardíaca, mesmo quando sabidamente por fibrilação ventricular, em apenas $70 \%$ dos casos se consegue indução de algum tipo de taquicardia ventricular no estudo eletrofisiológico invasivo, sendo que a taquicardia ventricular monomórfica sustentada corresponde somente a algo em torno de $40 \%$ das taquicardias induzidas ${ }^{21-29}$. Ainda, a incapacidade de induzir taquicardia ventricular não é indicativa de bom prognóstico, visto que os índices de recorrência de morte súbita nesses casos podem chegar a até $17 \%$ em 21 meses ${ }^{24}$.

Em síntese, um grande número de pacientes sobreviventes de parada cardíaca não apresenta taquicardias ventriculares inductíveis, apesar de permanecer em alto risco para um novo evento. E mais, nas situações de indução não é possível estabelecer uma relação causa-efeito entre aquela taquicardia induzida e o evento clínico. Se a aplicabilidade diagnóstica do estudo eletrofisiológico invasivo é questionável nessa população de indivíduos, seu emprego como método coadjuvante no tratamento é igualmente discutível. É sabido, de longa data, que a terapia farmacológica guiada por estimulação é capaz de reduzir a mortalidade em sobreviventes de parada cardíaca ${ }^{23,24,27}$. Na série de Wilber e cols. ${ }^{24}$, por exemplo, que envolveu 166 pacientes, a supressão da indução de qualquer forma de taquicardia ventricular pelo tratamento anti-arrítmico implicou um índice de mortalidade súbita de $12 \%$ em 21 meses de seguimento, contra $33 \%$ no grupo em que a supressão não foi obtida. Por mais que esses resultados sejam animadores, $12 \%$ de mortalidade arrítmica em pouco menos de dois anos persistem sendo um índice elevado e inaceitável. A situação é análoga quando se considera o tratamento ablativo de taquicardias ventriculares monomórficas induzidas. A eliminação de um ou mais substratos arritmogênicos não permite pressupor proteção contra a recorrência de parada cardíaca, particularmente devido não ser possível determinar o papel que esses substratos tiveram na gênese do evento clínico. Somam-se a isso os próprios índices de ineficácia das ablações, que são aguda e cronicamente elevados, o que leva alguns autores a recomendar implante de um cardioversor desfibrilador implantável, mesmo naqueles pacientes em que o procedimento eliminou todas as formas de taquicardias inductíveis, passíveis de mapeamento, visto que, mesmo nessas circunstâncias, os índices de recorrência de eventos arrítmicos em menos de um ano atingem até $20 \%{ }^{30}$.

\section{Considerações finais}

Com base nas evidências acumuladas, já discutidas, é notório que a utilidade do estudo eletrofisiológico invasivo em sobreviventes de parada cardíaca é no mínimo questionável, quando considerada na maioria das situações clínicas.

Frente a essas considerações, a realização de estudo eletrofisiológico invasivo seria de importância crucial apenas naqueles indivíduos, que sobreviveram a uma parada cardíaca não ligada a causas transitórias, onde a presença de uma doença cardíaca estrutural não seja estabelecida em uma avaliação criteriosa. A finalidade do estudo, nessas circunstâncias, seria a tentativa de identificar a presença de anormalidades cardíacas primariamente "elétricas", como uma síndrome de pré-excitação, uma taquicardia supraventricular, uma taquicardia ventricular idiopática, ou uma síndrome de Brugada inaparente.

Apesar de rotineira, a prática sistemática de estudo eletrofisiológico em sobreviventes de parada cardíaca não é cientificamente justificável, à luz do conhecimento atual. É óbvio que qualquer decisão médica não pode ser aplicada de maneira genérica, com base exclusivamente em resultados de estudos que expressam conclusões construídas com base em amostras uniformes de pacientes, quanto a suas características clínicas, com o intuito de dar respaldo a um cálculo estatístico. É bem conhecida na prática a dificuldade freqüente de enquadramento de uma situação clínica do dia-a-dia naquilo que efetivamente se estabeleceu por evidências. $\mathrm{O}$ princípio da individualização sempre foi e sempre será a base de uma atividade médica aplicada de uma forma correta. Por outro lado, a aplicabilidade de um determinado método diagnóstico ou terapêutico deve ser respaldada por uma base científica sólida, considerando-se sempre o "trinômio" risco-benefício-custo.

\section{Referências}

1. Zipes DP, DiMarco JP, Gillette PC, et al. ACC/AHA Task Force Report. Guidelines for Clinical Intracardiac Electrophysiological and Catheter Ablation Procedures. A Report of the American College of Cardiology/American Heart Association Task Force on Practice Guidelines (Committee on Clinical Intracardiac Electrophysiologic and Catheter Ablation Procedures). Developed in collaboration with the North American Society of Pacing and Electrophysiology. Published simultaneously in: J Cardiovasc Electrophysiol 1995; 6: 652-79. Circulation 1995; 92: 673-91. J Am Coll Cardiol 1995; 26: 555-73.

2. Sosa EA, Paola A, Gizzi JC, et al. Indicações para estudos eletrofisiológicos e ablação por cateter de arritmias cardíacas. Recomendações do DAEC da SBC. Arq Bras Cardiol 1995; 64: 149-51.

3. Böcker D, Block M, Borggrefe M, Breithardt G. Are electrophysiological studies needed before implantable cardioverter defibrillator surgery? Eur Heart J 1997; 18: $548-51$
4. Poole JE, Bardy GH. Sudden Cardiac Death. In: Zipes DP, Jalife J, eds. Cardiac Electrophysiology: from cell to bedside. Philadelphia: W.B. Saunders Co., 1995: 812-32.

5. Hallstrom AP, Eisenberg MS, Berner L. The persistence of ventricular fibrillation and its implication for evaluating EMS. Emerg Health Serv Q 1983; 1: 41-7.

6. Manolio TA, Furberg CD. Epidemiology of sudden cardiac death. In: Akhtar M, Myerburg RJ, Ruskin JN, eds. Sudden Cardiac Death. Prevalence, Mechanisms, and Approaches to Diagnosis and Management. Philadelphia: Williams \& Wilkins, 1994: 3-20.

7. Wever EFD, Hauer RNW, van Capelle FJL, et al. Randomized study of implantable defibrillator as first-choice therapy versus conventional strategy in postinfarct sudden death survivors. Circulation 1995; 91: 2195-203.

8. The antiarrhythmic versus implantable defibrillators (AVID) investigators: A 
comparison of antiarrhythmic-drug therapy with implantable defibrillators in patients resuscitated from near-fatal ventricular arrhythmias. N Engl J Med 1997; 337: 1576-83.

9. Connolly SJ, Gent M, Roberts RS, et al. Canadian implantable cardioverter defibrillator study (CIDS): a randomized trial of the implantable cardioverter defibrillator against amiodarone. Circulation 2000; 1297-302.

10. Kuck KH, Cappato R, Siebels J, Ruppel R. Randomized comparison of antiarrhythmic drug therapy with implantable defibrillators in patients resuscitated from cardiac arrest: the Cardiac Arrest Study Hamburg (CASH). Circulation 2000; 102: 748-54.

11. Bayes de Luna A, Coumel P, Leclercq JF. Ambulatory sudden cardiac death: mechanisms of production of fatal arrhythmia on the basis of data from 157 cases. Am Heart J 1989; 117: 151-9.

12. Panidis IP, Morganroth J. Sudden death in hospitalized patients: cardiac rhythm disturbances detected by ambulatory electrocardiographic monitoring. J Am Coll Cardiol 1983; 2: 798-805.

13. Myerburg RJ, Estes D, Zaman L, et al. Outcome of resuscitation from bradyarrhythmic or asystolic prehospital cardiac arrest. J Am Coll Cardiol 1984; 4: 1118-22.

14. Luu M, Stevenson WG, Stevenson LW, et al. Diverse mechanisms of unexpected cardiac arrest in advanced heart failure. Circulation 1989; 80: 1675-80.

15. Remole S, Hansen R, Benditt DG. Mechanisms of bradyarrhythmic sudden death. In: Akhtar M, Myerburg RJ, Ruskin JN, eds. Sudden Cardiac Death: Prevalence, Mechanisms, and Approaches to Diagnosis and Management. Philadelphia: Williams \& Wilkins, 1994: 407-15.

16. Klein GJ, Bashore TM, Sellers TD, et al. Ventricular fibrillation in the WolffParkinson-White syndrome. N Engl J Med 1979; 301: 1080.

17. Hays LJ, Lerman BB, DiMarco JP. Nonventricular arrhythmias as precursors of ventricular fibrillation in patients with out-of-hospital cardiac arrest. Am Heart J 1989; 118: 53-7.

18. Wang YS, Scheinman MM, Chien WW, et al. Patients with supraventricular tachycardia presenting with aborted sudden death: incidence, mechanisms, and long-term follow-up. J Am Coll Cardiol 1991; 18: 1711-9.

19. Weaver WD, Hill D, Fahrenbruch CD, et al. Use of automatic external defibrillator in the management of out-of-hospital cardiac arrest. N Engl J Med 1988; 319 : 661-6.
20. Cobb LA, Weaver WD, Fahrenbruch CE, et al. Community-based interventions for sudden cardiac death: impact, limitations, and changes. Circulation 1992; 85: I-98 - I-102

21. Roy D, Waxman HL, Kienzle MG, et al. Clinical characteristics and long-term follow-up in 119 survivors of cardiac arrest: relation to inducibility at electrophysiologic testing. Am J Cardiol 1983; 52: 969-74.

22. Skale BT, Miles WM, Heger JJ, et al. Survivors of cardiac arrest: prevention of recurrence by drug therapy as predicted by electrophysiologic testing or electrocardiographic monitoring. Am J Cardiol 1986; 57: 113-9.

23. Eldar M, Sauve MJ, Scheinman MM. Electrophysiologic testing and follow-up of patients with aborted sudden death. J Am Coll Cardiol 1987; 10: 291-8.

24. Wilber DJ, Garan H, Finkelstein D, et al. Out-of-hospital cardiac arrest: use of electrophysiologic testing in the prediction of long-term outcome. N Engl J Med 1988; 318: 19-24.

25. Freedman RA, Swerdlow CD, Soderholm-Difatte V, et al. Prognostic significance of arrhythmia inducibility or noninducibility at initial electrophysiologic study in survivors of cardiac arrest. Am J Cardiol 1988; 61: 578-82.

26. Adhar GC, Larson LW, Bardy GH, et al. Sustained ventricular arrhythmias: differences between survivors of cardiac arrest and patients with recurrent sustained ventricular tachycardia. J Am Coll Cardiol 1988; 12: 159-65.

27. Furukawa T, Rozanski JJ, Nogami A, et al. Time-dependent risk of and predictors for cardiac arrest recurrence in survivors of out-of-hospital cardiac arrest with chronic coronary artery disease. Circulation 1989; 80: 599-608.

28. Poole JE, Mathisen TL, Kudenchuk PJ, et al. Long-term outcome in patients who survive out-of-hospital ventricular fibrillation and undergo electrophysiologic studies: evaluation by electrophysiologic subgroups. J Am Coll Cardiol 1990; 16: 657-65.

29. Natale A, Sra J, Axtell K, et al. Ventricular fibrillation and polymorphic ventricular tachycardia with critical coronary stenosis: does bypass surgery suffice? J Cardiovasc Electrophysiol 1994; 5: 988-94.

30. Marchlinski FE, Gottlieb CD, Callans DJ, et al. Ablative therapy for ventricular tachycardia in chronic coronary artery disease. In: Dunbar SB, Ellenbogen K, Epstein AE, eds. Sudden Cardiac Death. Past, Present, and Future. New York: Futura, 1997: 199-224. 